\title{
On the electric field transient anomaly observed at the time of the Kythira $M=6.9$ earthquake on January 2006
}

\author{
A. Konstantaras ${ }^{1}$, J. P. Makris ${ }^{1}$, F. Vallianatos ${ }^{1}$, and M. R. Varley ${ }^{2}$ \\ ${ }^{1}$ Technological Educational Institute of Crete, Chania, Crete, 731 33, Greece \\ ${ }^{2}$ ADSIP Research Centre, Department of Technology, University of Central Lancashire, Preston, PR1 2HE, UK
}

Received: 10 July 2007 - Revised: 29 October 2007 - Accepted: 29 October 2007 - Published: 22 November 2007

\begin{abstract}
The study of the Earth's electromagnetic fields prior to the occurrence of strong seismic events has repeatedly revealed cases were transient anomalies, often deemed as possible earthquake precursors, were observed on electromagnetic field recordings of surface, atmosphere and near space carried out measurements. In an attempt to understand the nature of such signals several models have been proposed based upon the exhibited characteristics of the observed anomalies and different possible generation mechanisms, with electric earthquake precursors (EEP) appearing to be the main candidates for short-term earthquake precursors. This paper discusses the detection of a ULF electric field transient anomaly and its identification as a possible electric earthquake precursor accompanying the Kythira $M=6.9$ earthquake occurred on the 8 January 2006.
\end{abstract}

\section{Introduction}

This research work investigates signal characteristics related to the nature of the great ULF electrical transient anomaly and its possible identification as an electric earthquake precursor (EEP) accompanying the $M=6.9$ Kythira earthquake occurred on the 8 January 2006 in the inner part of the Southern Hellenic arc, with epicenter coordinates N $36.21^{\circ}$, E $23.41^{\circ}$ and a focal-depth of $69 \mathrm{~km}$. The ULF transient electrical anomaly was recorded by the Technological Educational Institute of Crete MVC-2DS recording station located at $\mathrm{N} 35.44^{\circ}, \mathrm{E} 24.05^{\circ}$, at a distance of $103 \mathrm{~km}$ from the epicentre of the main earthquake bearing $\mathrm{N} 34 \mathrm{~W}$.

The MVC-2DS recording station was designed and developed by the Institute of Terrestrial Magnetism, Ionosphere and Radio-wave Propagation, Saint Petersburg Filial (SPbF IZMIRAN), Russian Academy of Sciences, under

Correspondence to: A. Konstantaras

(akonstantaras@chania.teicrete.gr) the INTAS-99-1102 project titled "Study of the ULF electromagnetic phenomena related to earthquakes (SUPRE)". The MVC-2DS recording station (Hattori et al., 2004) has the capacity to measure three electric (telluric) components (dynamic range $\pm 2.5 \mathrm{~V}$ ) and uses a torsion photoelectric magnetometer (frequency range $\sim 15 \mathrm{~Hz}$, measurement range $\pm 5000 \mathrm{nT}$ ). The sampling frequency is $50 \mathrm{~Hz}$. The magnetic sensor is installed in the ground at $0.5 \mathrm{~m}$ depth and is covered with a plastic waterproof box. A 24 bit ADC unit is set up in a protective box in the vicinity of the magnetic sensor. $\mathrm{Pb}$ $\mathrm{PbCl}_{2}$ electrodes are buried in the ground at $1 \mathrm{~m}$ depth for telluric measurements forming an orthogonal couple of $100 \mathrm{~m}$ short dipoles deployed in the directions NS and EW, respectively. The ADC gets time stamp from a connected to it GPS and the collected data are recorded via an RS- 485 connection by the acquisition PC located in a shelter approximately $80 \mathrm{~m}$ away. The station has been operating since 2001 .

No foreshocks have been observed whilst there were only a few aftershocks considering the intermediate depth and the magnitude of the main event. The two subplots on Fig. 1 demonstrate the seismic sequence (Fig. 1a) in parallel with the observed electric transient anomaly (Fig. 1b). It is interesting to note that the swarm of the aftershocks practically ends with the return of the electric recordings to the background level (Fig. 1), although the main event occurred almost in the middle of the signal's duty cycle. On the other hand, we underline the preliminary fact of the absence of any significant magnetic signature, i.e. the particular transient anomaly accompanying the Kythira earthquake was solely observed with great amplitudes upon the recordings of the electric field (Figs. 2a and 3a) whilst there is no indication of the latter upon the equivalent recordings of the magnetic field (Figs. $2 \mathrm{~b}$ and $3 \mathrm{~b}$ ) besides a huge variation during the main shock due to the arrival of the elastic waves to the measuring site, which was filtered for presentation purposes. The vertical line on both subplots in Figs. 2 and 3 indicates the time of occurrence of the main seismic event.

Published by Copernicus Publications on behalf of the European Geosciences Union. 

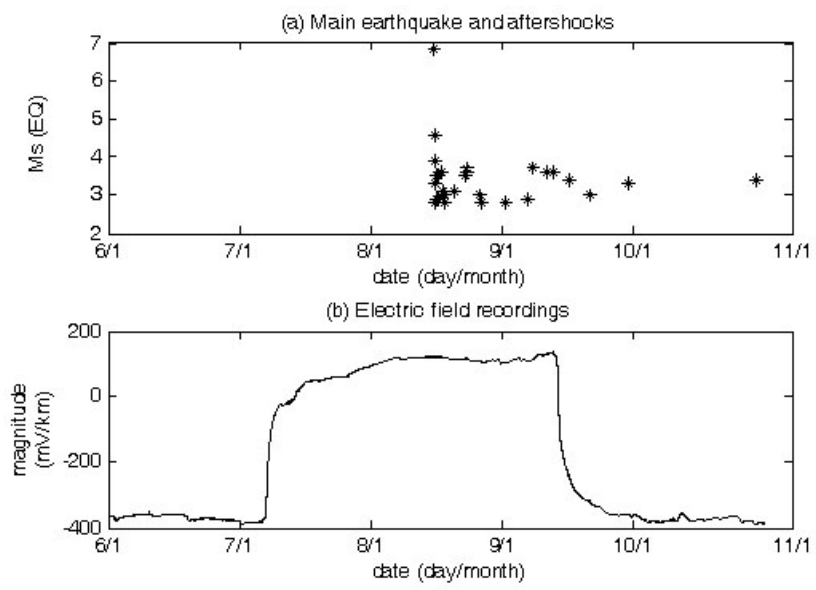

Fig. 1. (a) The position of the asterisks indicate the magnitude $(M s)$ and time of occurrence of the main earthquake and the following aftershocks. (b) The observed transient electric anomaly in parallel with the seismic sequence.
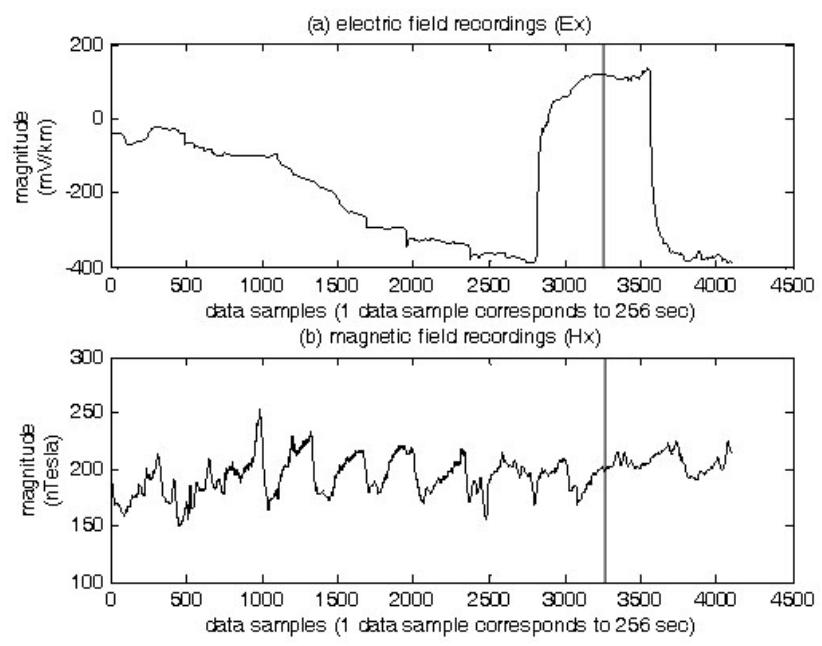

Fig. 2. (a) Recorded electric field signal (Ex - bearing N-S) from approximately 9 p.m. on the 29 December 2005 till 1 p.m. on the 11 January 2006. (b) Simultaneous magnetic field recordings ( $\mathrm{Hx}-$ bearing $\mathrm{N}-\mathrm{S}$ ). The vertical line indicates the time of the occurrence of the Kythira $M=6.9$ earthquake at approximately $11: 35$ a.m. on the 8 January, 2006.

The observed characteristics of the recorded possible electric earthquake precursor support previous results (Konstantaras et al., 2002, 2004, 2006a, b; Varotsos, 2005) regarding the features of EEP signals (Lighthill, 1996; Vallianatos and Tzanis, 1998; Tzanis and Vallianatos, 2001; Varotsos, 2005). It is well accepted that EEP signals are transient electric potential anomalies external to the natural electromagnetic field of the Earth (Hayakawa and Molchanov, 2002; Vallianatos and Tzanis, 1998; Konstantaras et al., 2002). To enhance and strengthen the detection and identification
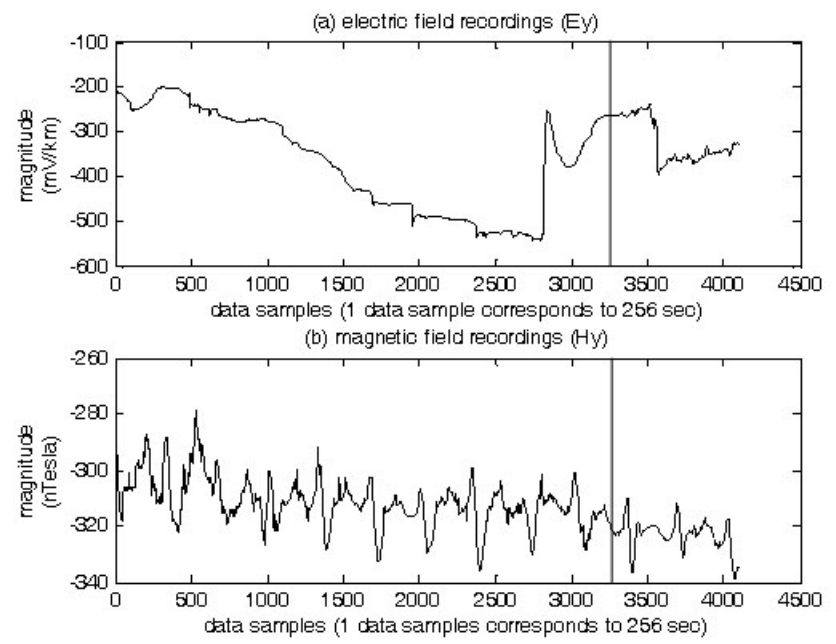

Fig. 3. (a) Recorded electric field signal (Ey - bearing E-W) from approximately 9 p.m. on the 29 December, 2005 till 1 p.m. on the 11 January, 2006. (b) Simultaneous magnetic field recordings (Hy bearing E-W). The vertical line indicates the time of the occurrence of the Kythira $M=6.9$ earthquake at approximately $11: 35 \mathrm{a} . \mathrm{m}$. on the 8 January, 2006.

of the observed transient electric field anomaly as an EEP signal associated with the Kythira EQ, the authors have employed a pattern recognition application with the incorporation of neuro-fuzzy technology. A neuro-fuzzy model has been trained to predict (Konstantaras et al., 2006a, b) the recorded electric field signal during time-periods of minimal seismic activity. Following successful training, the activation of the neuro-fuzzy model upon the electric field recordings around the time of the Kythira earthquake resulted in the rejection of the observed EEP signal from the surface electric field recordings. The neuro-fuzzy model has "decided" that the observed variation is not part of the natural electric field of the Earth due to ionospheric variability, thereby considerably suppressing the variation at its output.

\section{Possible EEP signals extrinsic to the natural electric field of the Earth}

The problem that still remains open and controversial is how to reliably discriminate earthquake-related signals from other man-made or natural noises. The difficulty of clear detection and undoubted identification of these signals obfuscates the understanding of their generation and propagation mechanisms. Several possible EEP generation mechanisms (Varotsos, 2005; Lighthill, 1996; Tzanis and Vallianatos, 2002) including solid state physics mechanisms, such as stress stimulating currents, charged dislocations and Lazarus model; and electrokinetic phenomena, state that EEP signals are the result of a different generation mechanism to the source that causes the natural electric field background. Furthermore, 
laboratory experiments (Freund, 2007; Hollerman, 2006; Varotsos, 2005), have shown that when rocks such as granite or gabbro are subjected to deviatoric stresses electronic charge carriers are activated. In addition the current flowing through the rocks in the unstressed portion also increases significantly. Stress relaxation causes the rocks to return to an electrically inactive state.

To investigate this case, which strengthens the identification of the observed signal as a transient anomaly associated with the seismic activity in the Kythira region, we have resolved to a pattern recognition experiment with the incorporation of soft computing technology. A neuro-fuzzy model, i.e. a neural network with intrinsic fuzzy logic abilities (Jang, 1993), has been developed and trained to identify the recorded electric field signal using the data recorded prior to the occurrence of the possible electric earthquake precursor. Then, propagating through the electric field recordings, the neuro-fuzzy model is used to forecast the next sample of the recorded signal based upon a number of previously recorded data. The purpose of the experiment is to identify whether the neuro-fuzzy model follows the detected EEP signal as if it was a part of the natural due to ionospheric variability electric field; or rejects it as an external distortion by considerably suppressing the EEP aiming for the actual value of the natural due to ionospheric variability electric field alone. The Ex component of the recorded electric field signal has been selected for training the neuro-fuzzy model because of the clear distinction between the ULF electric transient anomaly and the electric field background.

To train and evaluate the reaction of the neuro-fuzzy model, 4096 data samples of electric field recordings (Fig. 2a) have been selected (Konstantaras et al., 2004), corresponding approximately to the time-period starting at 9 p.m. on the 29 December 2005 and ending at 1 p.m. on 11 January 2006, which include the possible electric earthquake precursor. Although the sampling frequency of the recorded data is $f_{s}=1 \mathrm{~Hz}$, the overall data set has been decimated by a factor of 256 and filtered by a Chebyshev lowpass filter to prevent aliasing, for it is very costly in processing time (Kosko, 1991) to train a neural network with such a heavy workload. A sliding window consisting of four previous inputs, at $n-12, n-24, n-36$ and $n-48$ (Addison and Wermter, 2002), propagating though the time-series determines the input vectors to the neuro-fuzzy model. The first half of the input vectors, i.e. the first 2048 samples in the time-series, is used to train the neuro-fuzzy model to predict the next sample $(n+1)$ in the time-series (Jang et al., 1997), whilst the second half remains unseen. An initial neuro-fuzzy model is obtained by applying grid partitioning (Konstantaras et al., 2002) on the first half of the input data set. This initial model is subjected to training with a hybrid algorithm (Jang, 1993), a combination of the least squares method and the backpropagation algorithm. During a forward pass an input vector is fed to the neuro-fuzzy model and the least squares estimator is used to adapt its consequent

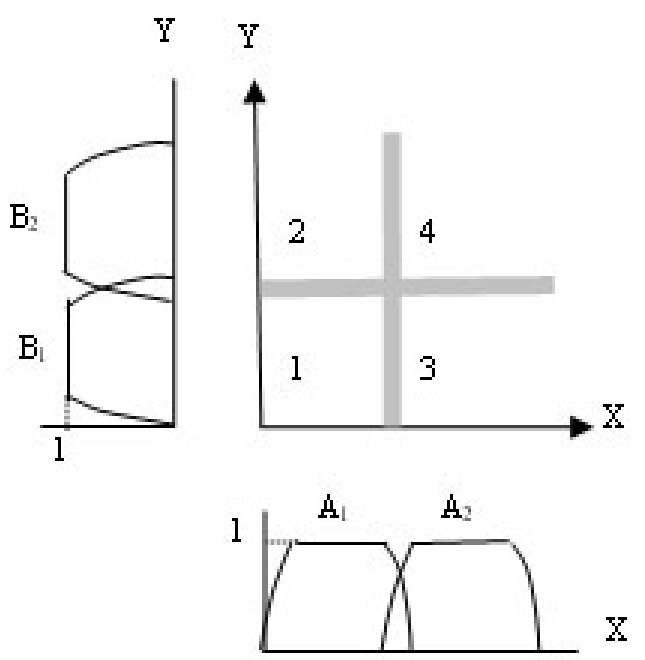

Fig. 4. Input space partitioning into four fuzzy regions corresponding to four fuzzy if-then rules. The number of inputs to the neurofuzzy model is four corresponding to an overall number of sixteen fuzzy if-then rules guiding the model.

parameters, which define the rules and output membership functions (MFs) of the model. A training error is computed by subtracting the output of the neuro-fuzzy model, for the current set of parameters, from the required output (the actual value of the electric field signal at $n+1$ ). The training error is deployed during the backward pass through the neuro-fuzzy model by the backpropagation algorithm to adapt its premise parameters, which determine the shape and dimensions of the input MFs. After every training epoch the neuro-fuzzy model is tested with the first 500 samples of the unseen data (also recorded before the occurrence of the possible electric earthquake precursor) to prevent overtraining (Jang et al., 1997). The final neuro-fuzzy model holds the parameters set that minimized the checking error.

\section{Neuro-fuzzy model architecture and operation analy- sis}

To generate an initial fuzzy inference system grid partitioning was applied on the input data of the input/output data set. For this particular application the two-dimensional input space of every input was partitioned into four overlapping fuzzy regions, each of which is governed by a fuzzy if-then rule (Fig. 4). The structure of the neuro-fuzzy model depends on the number of inputs and input membership functions per input. The developed neuro-fuzzy model, shown in Fig. 5, has four inputs (layer 1) each of which has assigned to it two input membership functions (layer 2) and is guided by sixteen rules (layer 3 ). The contribution of each rule to the output of the neuro-fuzzy model is determined by the output MF (layer 4) allocated to it, whilst the bias neuron (dashed 


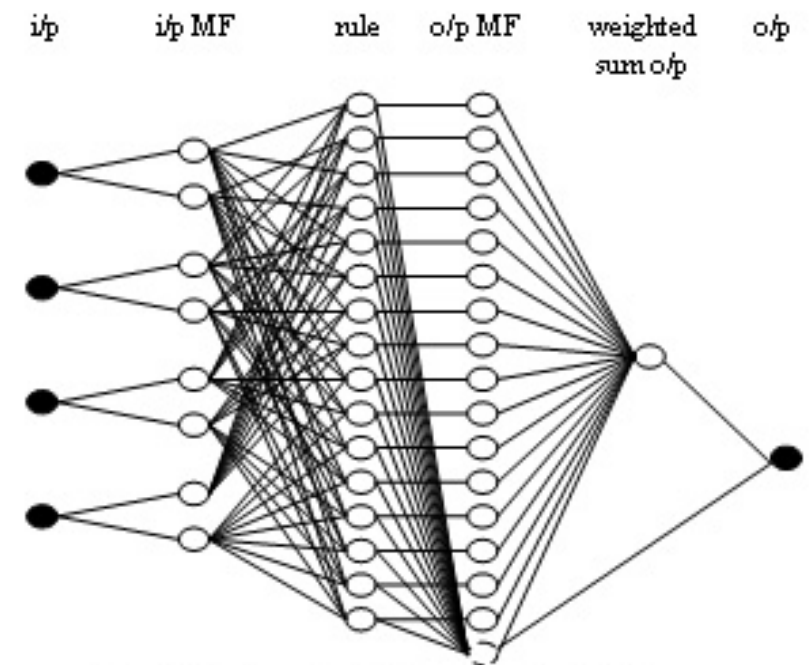

nodes - black: i'p - olp, white: neunons, dashed: bias neuron

Fig. 5. Neuro-fuzzy models' architecture. Black nodes: inputs to and output from the neuro-fuzzy model. White nodes: neurons. Dashed node: rules' bias neuron.

line in Fig. 5) sets a weighting factor to each rule. The neuron in layer 5 defuzzifies the normalised weighted outputs of all rules to produce a crisp output (layer 6). The layer by layer operation of the developed neuro-fuzzy model is described as follows (Jang, 1993):

Layer 1: The present and three previous samples of the recorded signal (electric or magnetic) are used as inputs ( $A$ to $D$ ) to the network introducing recursion.

Layer 2: Every node $i$ in this layer is an adaptive node with a node function: $O_{1, i}=\mu A_{i}(x)$, for $i=1,2$, or $O_{1, i}=\mu B_{i-2}(y)$, for $i=3,4$, or $O_{1, i}=\mu C_{i-4}(z)$, for $i=5,6$, or $O_{1, i}=\mu D_{i-6}(k)$, for $i=7,8$, where $x$ (or $y$, or $z$, or $k$ ) is the input to node $i$ and $A_{i}$ (or $B_{i}$, or $C_{i}$, or $D_{i}$ ) is the equivalent membership function. The type of MFs $A, B$, $C$ and $D$ is that of the generalised bell function: $\mu A(x)=$ $1 /\left(1+\left|\frac{x-c_{i}}{a_{i}}\right|^{2 b}\right)$, where $\left\{a_{i}, b_{i}, c_{i}\right\}$ are the premise parameters of the network which determine the shape and size of the MF.

Layer 3: Every node in this layer is a fixed node calculating the normalised firing strength of either rule: $O_{2, i}=\overline{w_{i}}=\frac{w_{i}}{\sum_{i} w_{i}}$, where $w_{i}=\mu A_{i}(x) \mu B_{i}(y) \mu C_{i}(z) \mu D_{i}(k)$.

Layer 4: Every node $i$ in this layer is an adaptive node using an output membership functions to compute the weighed output of the equivalent rule, according to the following node function: $O_{3, i}=\overline{w_{i}} f_{i}$ and $f_{i}=p_{i}+q_{i}+m_{i}+n_{i}+r_{i}$, where $\left\{p_{i}\right.$, $\left.q_{i}, m_{i}, n_{i}, r_{i}\right\}$ are the consequent parameters of the network that specify the rules of the fuzzy inference system.

Layer 5: The single node in this layer is a fixed node, which converts the weighted fuzzy outputs of all rules in the
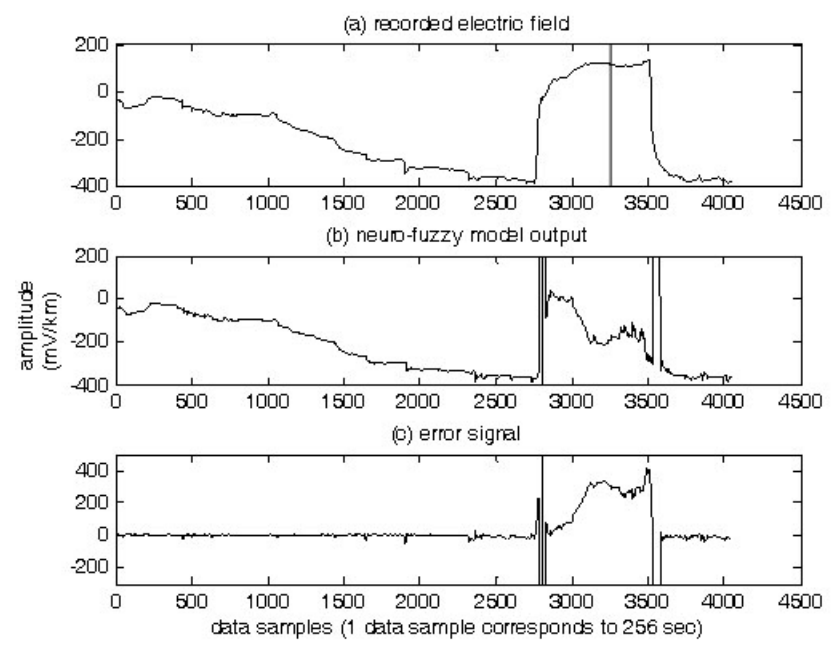

Fig. 6. (a) Recorded electric field signal - the vertical line indicates the time of the occurrence of the Kythira $M=6.9$ earthquake at approximately 11:35 a.m. on the 8 January, 2006; (b) Neuro-fuzzy model output indicating rejection of the possible EEP signal as an external addition upon the electric field recordings; (c) Error signal - the difference between the recorded electric field signal the output signal from the neuro-fuzzy model.

system into a single crisp output, as described by the following node function: $O_{4,1}=\sum_{i} \bar{w}_{i} f_{i}$.

Layer 6: The node describes the actual output of the neurofuzzy model for a given input data set.

\section{Pattern recognition results}

The proposed neuro-fuzzy model was initially trained upon the first 2548 data-samples, of recorded electric field signal shown in Fig. 2a aiming to identify the main characteristics of the electric field variations and thus predict the next sample in the time-series. The initial 2048 data samples were used for training the neuro-fuzzy model whilst the last 500 unseen to the network data samples (2049 to 2548) were used to monitor its performance and prevent overtraining the model.

The outcome of the neuro-fuzzy pattern recognition application on the full electric field signal after training the neurofuzzy model is shown in Fig. 6, where the vertical line on subplot "a" indicates the time occurrence of the main seismic event. Comparing subplots "a" and "b" on Fig. 6, there is an apparent significant suppression of the density of the possible recorded EEP signal. In detail, the output of neurofuzzy model closely follows the recorded electric field signal until the moment of the occurrence of the possible EEP at approximately data-sample 2752 . The rapid rise in magnitude of the recorded signal over the next few samples "confuses" the neuro-fuzzy model and for a short time it becomes unstable, hence the large spikes observed between data-samples 
2780 and 2830. Thanks to the adaptive nature (Haykin, 1994) of neural networks only a short time of approximately fifty samples was required by the neuro-fuzzy model for it to react to the new information received at its input. Following data-sample 2830 the neuro-fuzzy model makes a decision not to follow the information received at its input, i.e. the signal shown by subplot "a" on Fig. 6, when deciding what should be the value of the next sample in the time-series at its output. Instead it tries to approximate the magnitude of the natural electric field alone, hereby considerably suppressing the possible recorded EEP signal. Around data sample 3510 the neuro-fuzzy is affected by a sudden drop in the magnitude data received at its input (ending of the possible EEP signal). Once-more the neuro-fuzzy model temporarily becomes unstable and hence the large spikes observed between data-samples 3530 and 3585 . What is remarkable in this case is that once the model becomes stable again, which is almost immediately after the end of the duration of the EEP signal around data sample 3595, the neuro-fuzzy model follows closely once more the recorded electric field signal.

The fact that the neuro-fuzzy model follows closely the recorded electric field signal before (signal to difference ratio of $30.46 \mathrm{~dB}$ ) and after (signal to difference ratio of $28.40 \mathrm{~dB}$ ) the occurrence of the EEP signal, and the rejection of the latter (signal to difference ratio of $-39.69 \mathrm{~dB}$ ) at the time of its occurrence (Fig. 6c) makes us believe that the neuro-fuzzy model treats the EEP signal as an external distortion added upon the natural due to ionospheric variability electric field of the Earth.

\section{Conclusions}

The possible electric earthquake precursor accompanying the Kythira $M=6.9$ earthquake was detected upon the electric field recordings of the electromagnetic field recording station in Keramia, Crete, but with no indication of the latter upon simultaneous magnetic field measurements. The outcome of the neuro-fuzzy pattern recognition experiment, suggests that this particular EEP-candidate signal is an extrinsic transient electric potential anomaly of a different source to the natural and of ionospheric origin electric field of the Earth. The error signal highlights the close proximity of the neuro-fuzzy model's output signal to the recorded electric field before and after the occurrence of the possible recorded EEP signal as well as the continuous incremental rejection of the latter at the time of its occurrence. The neuro-fuzzy model was also successful in estimating the boundaries of the observed transient electric potential anomaly which are in accordance with the observed data. The duration of the possible EEP signal, outlasts other detected EEP candidate signals, lasting for a couple of days rather than a couple of hours. Furthermore, the possible EEP signal attributed to the Kythira earthquake precedes but also super-cedes the main seismic event which occurred at 11:34 GMT on the 8 January, 2006. The rising edge of the EEP signal occurred almost $36 \mathrm{~h}$ prior the main seismic event around 01:00 GMT on 7 January 2006. The EEP though outlasted the main seismic event with its falling edge occurring approximately $25 \mathrm{~h}$ later, around 13:00 GMT on the 9 January 2006.

Acknowledgements. This work is supported by the project Archimedes I: "Support of Research Teams of Technological Educational Institute of Crete", MIS 86384, sub-project 2.2.15 entitled "Multiparametric Spatiotemporal Estimation of Seismic Hazard in the Front of the Hellenic Arc. Application to the Protection of Old City-Venetian Harbor of Chania, Crete, Greece", Action 2.2.3. $\zeta$, in the frame of Operational Programme for Education and Initial Vocational Training (O.P. "Education") one of the Third Community Support Framework's 24 Operational Programmes (2000-2006) in Greece, and is co-financed by the European Social Fund $(75 \%)$ and national resources $(25 \%)$.

Edited by: P. F. Biagi

Reviewed by: two anonymous referees

\section{References}

Addison, J. F. D. and Wermter, S.: Methods for investigating memory into neural networks applied to condition monitoring, Proc. of the Int. Conf. on A.I. and Soft Computing, Banff, Alberta, Canada, 380-384, 2002.

Freund, F. T.: Pre-earthquake signals - Part I: Deviatoric stresses turn rocks into a source of electric currents, Nat. Hazards Earth Syst. Sci., 7, 535-541, 2007, http://www.nat-hazards-earth-syst-sci.net/7/535/2007/.

Hattori, K.: ULF geomagnetic field measurements in Japan and some recent results associated with Iwateken Nairiku Hokubu earthquake in 1998, Phys. Chem. Earth, 29, 481-494, 2004.

Hayakawa, M. and Molchanov, O. A.: Seismo Electromagnetics Lithosphere-Atmosphere-Ionosphere Coupling, TERRAPUB, Tokyo, 335-343, 2002.

Haykin, S.: Neural networks: a comprehensive foundation, Macmillan College Publishing, 135-155, 1994.

Hollerman, W.: Electric currents in granite and gabbro generated by impacts up to $1 \mathrm{~km} / \mathrm{sec}$, AGU Fall Meeting, San Francisco, T31A-0419, 2006.

Jang, J.: ANFIS: Adaptive Network-based Fuzzy Inference Systems, IEEE Transactions on Systems, Man, and Cybernetics, 23(3), 665-685, 1993.

Jang, J., Sun, T., and Mizutany, E.: Neuro-fuzzy and soft computing, Prentice Hall, 335-363, 1997.

Konstantaras, A., Varley, M. R., Vallianatos, F., Collins, G., and Holifield, P.: Recognition of electric earthquake precursors using neuro-fuzzy methods: methodology and simulation results, Proc. IASTED Int. Conf. Signal Processing, Pattern Recognition and Applications (SPPRA 2002), Crete, Greece, 303-308, 2002.

Konstantaras, A., Varley, M. R., Vallianatos, F., Collins, G., and Holifield, P.: A neuro-fuzzy approach to the reliable recognition of electric earthquake precursors, Nat. Hazards Earth Syst. Sci., 4, 641-646, 2004, http://www.nat-hazards-earth-syst-sci.net/4/641/2004/.

Konstantaras, A., Varley, M. R., Vallianatos, F., Collins, G., and Holifield, P.: Neuro-fuzzy prediction-based adaptive filtering 
applied to severely distorted magnetic field recordings, IEEE Geosci. Remote S., 3(4), 161-165, 2006 a.

Konstantaras, A., Varley, M. R., Vallianatos, F., Collins, G., and Holifield, P.: Neuro-fuzzy technology for the detection of weak seismo-electric signals, Proc. of the Atlantic Europe Conference in Remote Imaging and Spectroscopy (AECRIS), Preston, UK, 151-157, 2006b.

Kosko, B.: Neural networks for signals processing, Prentice Hall, Upper Saddle River, NJ, 399 pp., 1991.

Lighthill, J.: A critical review of VAN, World Scientific, Singapore, 29-76, 1996.

Teisseyre, R. (Ed.): Theory of Earthquake premonitory and fracture processes, Polish Scientific Publishers, 520-528, 1995.

Teisseyre, R. and Nagahama, H.: Dislocational models of electric field generation in a seismic source zone, in: Atmospheric and Ionospheric Electromagnetic Phenomena Associated with Earthquakes, edited by: Hayakawa M., Terra Scientific Publishing, Tokyo, 271-285, 1999.
Tzanis, A. and Vallianatos, F.: A critical review of Electric Earthquake Precursors, Annali di Geofisica, 44(2), 429-460, 2001.

Tzanis, A. and Vallianatos, F.: A physical model of electric earthquake precursors due to crack propagation and the motion of charged edge dislocations, in: SeismoElectromagnetics: Lithosphere-Atmosphere-Ionosphere Coupling, edited by: Hayakawa, M. and Molchanov, O. A., Terra Sci. Pub. Co., Tokyo, 117-130, 2002.

Vallianatos, F. and Tzanis A.: Electric current generation associated with the deformation rate of a solid: Preseismic and coseismic signals, Phys. Chem. Earth, 23, 933-938, 1998.

Varotsos, P. A.: The physics of seismic electric signals, edited by: Terrapub, Tokyo, 251-272, 2005. 